\title{
La polémique autour des « délits de la pensée » dans le contexte de l'autoritarisme vietnamien : un processus de démocratisation?
}

The controversy on "crimes of thought" in the context of Vietnamese authoritarianism: a process of democratization?

Thi Thanh Phuong Nguyen-Pochan

\section{(2) OpenEdition}

\section{Journals}

Electronic version

URL: http://journals.openedition.org/aad/5256

DOI: $10.4000 /$ aad. 5256

ISSN: 1565-8961

\section{Publisher}

Université de Tel-Aviv

\section{Electronic reference}

Thi Thanh Phuong Nguyen-Pochan, "La polémique autour des « délits de la pensée » dans le contexte de l'autoritarisme vietnamien : un processus de démocratisation ?", Argumentation et Analyse du Discours [Online], 26 | 2021, Online since 12 April 2021, connection on 16 April 2021. URL: http:// journals.openedition.org/aad/5256 ; DOI: https://doi.org/10.4000/aad.5256

This text was automatically generated on 16 April 2021.

\section{cc) $(1) \odot$}

Argumentation \& analyse du discours est mis à disposition selon les termes de la licence Creative Commons Attribution - Pas d'Utilisation Commerciale - Pas de Modification 4.0 International. 


\title{
La polémique autour des « délits de la pensée » dans le contexte de l'autoritarisme vietnamien : un processus de démocratisation ${ }^{1}$
}

\author{
The controversy on "crimes of thought" in the context of Vietnamese \\ authoritarianism: a process of democratization?
}

Thi Thanh Phuong Nguyen-Pochan

Fin 2018, le Parti communiste vietnamien (PCV) a désaffilié Chu Hảo, un de ses intellectuels, à titre de sanction. Ancien Ministre adjoint des Sciences et des Technologies et Directeur de la Maison d'édition Savoir, il est une des figures éminentes de l'intelligentsia vietnamienne engagée dans la voie de la démocratisation du pays. Il fut accusé par le Comité central de l'inspection du PCV (désormais «le comité »), le 25 octobre 2018, de "dégénérescence de la pensée politique, d"“auto-évolution" et d"“auto-transformation" ». Cette sanction a suscité un étonnement puis un vif tollé chez les intellectuels qui y ont vu une attaque contre leur milieu. Une polémique a ainsi émergé sur Internet qui s'est déroulée pendant les trois derniers mois de 2018.

2 Dans son ouvrage Apologie de la polémique (2014), Ruth Amossy a montré que la polémique, loin d'être un phénomène négatif, permet une coexistence des opinions divergentes dans un espace pluraliste là où la délibération en vue d'un consensus échoue régulièrement. Selon l'auteure, la polémique est une modalité argumentative intrinsèque à toute communication en régime démocratique, car le conflit est la règle dans l'espace pluraliste; l'importance est de comprendre comment cette " rhétorique du dissensus » fonctionne et participe au débat public (ibid. : 15). Si la polémique est un facteur indispensable au bon fonctionnement de la démocratie, elle pourrait être, selon notre hypothèse, un révélateur de démocratisation de l'espace public en régime autoritaire. 
3 Dans la polémique concernant l'affaire de Chu Hảo, nous n'entrerons pas dans tous les sujets complexes débattus mais nous focalisons uniquement sur les accusations (explicitées par la suite) d'" auto-transformation» et d'" auto-évolution» qui apparaissent comme les discours «les plus menaçants " (Maingueneau $1984: 122$ ) et génèrent une redondance d'énoncés. Nous allons décrire et analyser les modalités discursives de cette polémique qui rendraient possible une «coexistence dans le dissensus » (Amossy 2014) en régime autoritaire.

Notre étude s'appuiera sur un corpus constitué des occurrences d'information couvrant cette affaire pendant les trois derniers mois de 2018 et qui sont issues de deux sources. D'un côté, nous avons onze occurrences prises dans les six médias qui représentent la voix officielle de l'État-parti ${ }^{2}$ : un article du portail électronique du comité, un article du quotidien Le Peuple, trois articles du journal L'Armée du Peuple, deux articles du journal Police du Peuple, un article du journal Le Travailleur et trois reportages du JT de 19 heures de la Télévision vietnamienne (VTV). De l'autre côté, 23 occurrences issues de quatre médias sociaux populaires ${ }^{3}$ auprès de l'intelligentsia vietnamienne: Bauxite Vietnam (BVN, 12 articles), Journaliste citoyen (trois articles), La voix du peuple (cinq articles) et Viet-Studies (trois articles); et huit entrées issues des pages Facebook.

Cette polémique ayant suscité un grand nombre de prises de parole, nous ne pouvons traiter de manière exhaustive tous les discours qui y participent sur Internet. Notre sélection du corpus est basée sur la légitimité et la représentativité de l'énonciateur, en réponse aux questions de savoir qui parle et au nom de qui il parle: d'un côté, les médias officiels retenus sont les représentants de la voix de l'État-parti; et de l'autre côté, les énonciateurs sur Internet dont l'autorité est reconnue par les intellectuels vietnamiens, cela leur permet de représenter les intellectuels attaqués et de parler en leur nom dans une affaire qui les concerne. Dans le contexte particulier de l'autoritarisme vietnamien et de sa récupération des paroles contestataires à ses fins politiques, notre analyse cherche à éclaircir les procédures de polarisation et le processus dialogique qui organisent la coprésence des discours antagonistes dans l'espace public, mais aussi la fracture issue du camp des intellectuels contestataires.

\section{L'autoritarisme vietnamien et l'existence de la polémique}

\subsection{La transformation de l'autoritarisme post-réforme}

6 Avant le lancement des réformes socio-économiques du Vietnam ${ }^{4}$, les critiques circulaient dans des cercles restreints ou étaient réduites au silence par le pouvoir. Bien que les voix contestataires n'aient jamais cessé d'exister au Vietnam sous le régime communiste ${ }^{5}$, elles n'eurent pourtant aucune visibilité au regard de la majorité de la population. L'arrivée d'Internet à la fin des années 1990 a fortement modifié le régime de visibilité de ces voix marginales, en leur donnant les possibilités de s'exprimer et de participer à la reconfiguration profonde de l'espace public.

$7 \quad$ Le $\mathrm{VI}^{\mathrm{e}}$ congrès national du PCV (1986) a donné le feu vert à la presse pour qu'elle puisse s'exprimer un peu plus librement. Il a fait naître une presse dite "révolutionnaire critique " servant à propager le mythe d'un parti déterminé et transparent dans sa lutte contre la corruption, la bureaucratisation, les fléaux issus de l'économie de 
marché et la traque aux éléments aliénés au sein du parti (Hữu 1998 : 459-461). Depuis le $\mathrm{X}^{\mathrm{e}}$ congrès du PCV (2006), le concept de " critique sociale » est officiellement apparu dans le langage politique, désignant les avis issus de la société civile qui participent aux débats autour des projets socio-économiques mis en place par l'État-parti (Trần 2006). Le Parti a assigné au Front de la patrie un rôle central dans le processus de collecte de ces avis : représentant du Parti en matière de rassemblement des masses, le Front contrôle les organisations de masse, surveille et canalise leurs critiques. Ces « organisations de façade » (Arendt 1972) servent d'intermédiaire entre le Parti et le peuple en fédérant les publics différents sous la direction unique du Parti (organisations de femmes, de vétérans ou d'agriculteurs, unions d'écrivains, de journalistes ou de jeunes communistes, etc.). Elles ont pour mission d'assurer la «solidarité du peuple », c'est-àdire de faire de la propagande auprès du peuple et de surveiller des opinions hostiles et des symptômes de " déviation idéologique ».

Le concept de critique sociale emprunté au lexique de la démocratie moderne (État de droit, société civile, discussion, rationalité, droit démocratique, liberté d'expression) est censé servir d'outil pour promouvoir le débat social et établir des liens entre les institutions politiques et la société civile (Phan 2015: 59). Cependant, l'État-parti conserve son rôle de maître du jeu en dictant la limite de la critique sociale (sont exclues les opinions contre le régime et le Parti), et en décidant des personnes autorisées à participer à ce processus, à savoir les membres des organisations de masse reconnues par l'État-parti. De plus, les critiques doivent être "constructives»: Or, discerner la frontière floue qui sépare critique constructive de critique destructive n'est pas une mince affaire, même pour les médias officiels du régime. L'expérience de ces dernières décennies montre que la presse a subi des sanctions à plusieurs reprises sous le prétexte de critique destructive; en réalité, elle a été victime des conflits internes au sein de l'État-parti (Papin et Passicousset 2010, Cain 2013).

9 Le feu vert donné à la critique sociale pourrait être interprété comme un effort du régime pour faire face à des tensions nouvelles nées du contexte de réforme. La libéralisation de la vie socio-économique exerce un fort impact sur la libéralisation de la pensée politique. Depuis les années 2010, l'émergence d'une société civile, corrélée à l'arrivée d'Internet, a donné lieu à une multiplication d'organisations indépendantes et de revendications politiques (pluralisme, multipartisme, droits de l'homme, liberté d'expression...) dont les voix les plus audibles se font entendre sur les plateformes numériques. Face à cette nouvelle conjoncture socio-politique, le régime vietnamien, tout comme son homologue chinois, devait modifier ses méthodes de gouvernance : celles-ci sont désormais caractérisées par une combinaison arbitraire de fermeté et souplesse, ou de répression et tolérance, laquelle se traduit en des attitudes ambiguës et des mesures de contrôle souvent imprévisibles et indéchiffrables (London 2014).

La relative tolérance vis-à-vis des voix contestataires fait partie de l'ensemble des dispositifs de contrôle déployés par le régime pour mieux les surveiller, pour censurer et canaliser l'opinion publique. Comme le remarque Fiskesjö (2017:1) dans son étude sur l'autoritarisme chinois, lorsque la foi en l'idéologie communiste s'est perdue, le régime est entré dans l'ère de post-vérité : afin de fabriquer sa propre vérité, il a publicisé les voix dissidentes pour les stigmatiser et humilier dans l'espace public, en les présentant comme opposées à l'intérêt national et nuisibles à une société harmonieuse. L'autoritarisme chinois se sert également de la polémique pour brouiller les protestations ou dénoncer publiquement toutes accusations portées contre lui 
comme fake news. Ainsi, faire entendre le discours contestataire vise principalement à le stigmatiser (réactionnaires, éléments subversifs, opportunistes...). Cependant, cela permet aussi, volens nolens, d'en faire des opinions qui participent au débat public.

\subsection{L'impact de la polémique sur l'espace public en régime autoritaire}

11 Pour les autorités, il ne s'agit donc pas d'une démocratisation souhaitée parce que jugée légitime, mais bien d'une manœuvre pragmatique estimée nécessaire. Dans l'espace public numérique où la libéralisation de la pensée politique semble inévitable, les autorités laissent les contestations s'expriment pour mieux les contrôler. La manipulation politique de la polémique montre à la fois la fonction que le régime lui attribue et les limites de cette récupération des contestations en sa faveur. Il n'en reste pas moins que la polémique en régime autoritaire - c'est notre hypothèse - pourrait, dans une certaine mesure et malgré la volonté des autorités, avoir certaines fonctions socio-discursives semblables à celles qu'elle remplit en régime démocratique - en particulier, protester contre l'Autre, inciter à l'action, influencer un tiers ou tisser du lien social (Amossy 2014); elle pourrait contribuer ainsi à la démocratisation de l'espace public.

Dans la polémique qu'on étudie ici, les voix contestataires proviennent principalement des intellectuels réformistes, ceux qui ont servi l'État-parti pendant longtemps en tant que hauts fonctionnaires (généraux de l'armée, membres du gouvernement, écrivains, professeurs ou chercheurs...). Ces dernières années, ils ont peu à peu quitté l'État-parti pour rejoindre les organisations indépendantes issues de la société civile et mené des protestations contre le régime communiste. Ils revendiquent une réforme totale du système pour démocratiser la vie politique et choisissent la coopération et le dialogue avec le pouvoir afin d'installer progressivement la démocratie. Leur revendication fait émerger une figure d'intellectuel qualifiée par Thayer (2006: 118) d'« opposants fidèles ». Cependant, leur position de insider a été régulièrement critiquée par les intellectuels dissidents comme celle de " pro-orthodoxie » (Phạm 2009) : à leurs yeux, la critique des opposants fidèles ne sert qu'à obtenir un "compromis qui protège et renforce le statu quo » (Phạm 2012). Pendant la période de la polémique, les réformistes et les dissidents se sont ralliés contre la sanction du PCV. Mais le lien organique des intellectuels réformistes avec le PCV ainsi que la fracture au sein des intellectuels contestataires persistent et interviennent au cours de la confrontation.

\subsection{La polémique autour des « délits de la pensée »}

13 Les appellations d'«auto-évolution» et d'«auto-transformation » relèvent de la «formule» dans le discours politique, désignant «l'ensemble de formulations qui, du fait de leurs emplois à un moment donné et dans un espace public donné, cristallisent des enjeux politiques et sociaux " (Krieg-Planque 2009: 7). Leur usage remonte à une notion plus ancienne qu'est l'« évolution pacifique». «Le terme d'évolution pacifique est utilisé pour la première fois par la Chine à la fin des années 1970, qui a été emprunté par le Vietnam pour décrire la stratégie de l'impérialisme [occidental] visant à miner le socialisme en Europe de l'Est et Union soviétique»(Thayer 1999: 14). Depuis 
l'effondrement de l'Union soviétique en 1991, l'évolution pacifique est devenue une obsession des idéologues conservateurs du PCV (ibid. : 15).

L'auto-transformation et l'auto-évolution, dérivés du terme d'évolution pacifique, désignent "l'évolution interne du parti dans une direction différente de celle qui est dictée et imposée par le Politburo sous l'effet des influences pro-démocratiques extérieures. Elle fonctionne donc comme un avertissement au sein de l'appareil politique et militaire" (Guillemot 2016). Si tous ces trois termes représentent la menace «souple» (saper le régime par la voie économique ou culturelle), l'évolution pacifique se tourne vers l'ennemi du dehors alors que l'auto-évolution et l'autotransformation se tournent vers l'ennemi du dedans (cadres, hauts fonctionnaires, militaires, etc. de l'État-parti) qui pourrait se transformer en "agent politique de l'Occident » (ibid.). Depuis l'arrivée du secrétaire général conservateur Nguyễn Phú Trọng en 2011, la ligne directrice du PCV s'est durcie et s'est officialisée à travers la Résolution $4^{6}$, conçue comme une mesure urgente face aux mutations de la société civile et surtout aux revendications de réformes issues des intellectuels de l'establishment.

\section{Les discours polémiques antagonistes}

\subsection{Positionnement et enjeu définitoire}

La polémique a commencé le 25 octobre 2018, lorsque le comité a annoncé sa conclusion sur « la dégénérescence de la pensée politique, "l'auto-évolution" et "l'autotransformation" » de Chu Hảo. Elle s'est intensifiée avec la décision de "désaffiliation du Parti », annoncée le 15 novembre 2018. Dans l'article du 31 octobre 2018, le comité a mis en avant quatre « délits » attribués à Chu Hảo: en tant que directeur de la maison d'édition Savoir, « il a édité et publié des ouvrages dont les contenus sont contraires à la ligne directrice du PCV »; « rédigé et signé des lettres ouvertes et des pétitions en vue de protester contre le régime et de proposer une réforme totale du système "; "échangé avec les médias étrangers et publié des contenus erronés sur les médias sociaux »; "créé et participé à plusieurs organisations de la société civile afin de propager des pensées erronées ${ }^{7}$.

Le quotidien Le Peuple et le journal L'Armée du peuple, deux médias pivots du régime, ont immédiatement présenté leurs arguments pour défendre les accusations du comité. Ils font appel à des arguments d'autorité - définis comme des arguments « en faveur d'une affirmation» qui reposent, non sur un examen de sa justesse mais sur le fait qu'elle " ait été énoncée par un locuteur particulier autorisé " qui est "garant de sa justesse " (Plantin 1996 : 88). Ces médias s'appuient sur les documents officiels de l'État-parti tels que la loi sur l'édition de 2012, les règles 47 (2011), 101 (2012) et 102 (2017) du comité central du PCV concernant les interdictions et les formes de sanction pour les membres du PCV. Selon Le Peuple (29/10/2018), ces documents montrent «le principe selon lequel les membres doivent respecter la discipline du parti et accepter ses formes de sanction correspondant au niveau de violation. " L'Armée du peuple (11/11/2018) se réfère à la Résolution 4 qui définit les «neuf symptômes de l'“auto-transformation et de l'auto-évolution" " :

En étant confronté à ses actes, monsieur chu Hảo a présenté les symptômes suivants : 1- Rejeter le marxisme-léninisme et la pensée de Ho Chi Minh et les règles 
$\mathrm{du}$ parti [...] et revendiquer «le pluralisme et le multipartisme »; 2- Rejeter le socialisme démocratique, l'État de droit socialiste, revendiquer la séparation des trois pouvoirs, le développement de la société civile, rejeter l'économie de marché à orientation socialiste et le régime foncier de la propriété collective; 3- Parler, écrire, agir à l'encontre de la ligne directrice et de la politique du parti et de l'État, etc. désignent un déraillement par rapport à la direction du parti. Ils agissent en tant qu'insider du système lorsqu'ils accusent Chu Hảo en tant que membre du parti et présentent la sanction du PCV comme une affaire interne. L'enjeu consiste à réfuter l'accusation d'« attaque contre les intellectuels» issue du camp adverse par une redéfinition qui recadre la question. L'Armée du Peuple (11/11/2018) affirme que «sanctionner un membre qui a commis des fautes n'est pas un traitement discriminatoire contre le groupe [intellectuel] ». Dans sa rubrique «Lutter contre l'évolution pacifique ", Police du peuple (18/11/2018) a commenté :

Ce n'est pas parce que monsieur Chu Hảo fait partie du milieu intellectuel que nous pouvons amalgamer la sanction du parti à l'encontre d'un membre qui a commis des fautes avec le traitement discriminatoire envers le milieu intellectuel [...] Monsieur Chu Hảo ne représente pas la classe intellectuelle vietnamienne.

Dans le camp adverse, il s'agit incontestablement d'une attaque contre les intellectuels dans la mesure où les formules d'accusation visent clairement à incriminer les activités intellectuelles dont celles de Chu Hảo offrent un exemple. Si, pour le PCV, ces délits sont destructeurs pour sa stabilité interne, aux yeux des contestataires, ils sont constructifs pour la société car ils sont du côté du progrès et de l'évolution de la société tout court.

Trần Trung Đạo, Facebook, 27/10/2018 : Le PCV accuse monsieur Chu Hảo d'autoévolution et d'auto-transformation, mais il oublie que l'évolution et la transformation constituent des processus naturels de l'homme [...] Mêmes les partis communistes [...] se sont auto-transformés en permanence au travers de ce qu'ils appellent « les réformes » pour leur survie.

Cao Huy Thuần, Viet-studies, 30/10/2018: Un seul mot dans ce procès suffit à montrer la mentalité arriérée [...], sans la transformation l'homme resterait un esclave. Et la société ne saurait se transformer si l'homme ne transforme pas son esprit [...] Pourquoi la publication des ouvrages qui ont marqué les grandes étapes de la pensée humaine est-elle un délit ? Dans quelle époque vit-on?

Nguyễn Đình Cống, BVN, 25/11/2018 : L'important n'est pas de lutter contre l'autoévolution, mais de créer une évolution qui est conforme à la loi du progrès.

L'enjeu de la re-sémantisation de ces formules réside dans l'affirmation selon laquelle ces prétendus délits de la pensée «ne servent qu'à l'intérêt égoïste du parti » (Hoa Nghi, BVN, 28/11/2018), laissant de côté l'intérêt du pays car ils sont contraires au développement et au progrès. En restituant son sens positif au terme d'évolution, les intellectuels se positionnent comme les défenseurs des valeurs progressistes et démocratiques. Pour eux, la sanction contre Chu Hảo n'est pas une simple affaire interne du parti : elle porte atteinte à l'ensemble des intellectuels et à la nation tout entière.

21 Leur divergence avec le PCV est donc irréconciliable. Ils estiment vivre dans une autre époque que celle du PCV - caractérisée par eux comme celle de la « pensée sauvage du moyen âge [...] au beau milieu de l'ère de la globalisation et de l'industrie 4.0 » (Lê Phú 
Khải, $B V N, 12 / 11 / 2018)$ ou celle de "l'Église romaine catholique au $17^{e}$ siècle qui a condamné Galilée » (Phạm Đình Trọng, BVN, 27/11/2018). Ils se réfèrent à une autre source d'autorité que celle du parti par le biais des citations des grands intellectuels de l'Occident, montrant ainsi leur adhésion volontaire aux valeurs et aux modes de réflexion des intellectuels éclairés de l'Occident :

Phạm Xuân Nguyên, Facebook, 26/10/2018: "Every opinion based on scientific criticism I welcome" (Karl Marx) [...] Souvenez-vous des propos de Christophe Colomb ?: "En suivant la lumière du soleil, nous avons laissé derrière nous l'Ancien Monde". Une fois que l'esprit est éclairé, on ne peut plus revenir aux ténèbres, comme le disait Thomas Paine.

Hoàng Dũng, BVN, 26/10/2018: Comme le disait Bernard Shaw, "Progress is impossible without change, and those who cannot change their minds cannot change anything."

Selon Nhân Trần ( $L a$ voix du peuple, 29/10/2018), les délits décrits constituent « une poignée de concepts insignifiants [...] Même ceux qui les écrivent ne comprennent pas leur sens sauf cette suggestion systématique: LE PARTI A TOUJOURS RAISON.» Le recours à l'argument d'autorité est ainsi rejeté comme celui à la pensée dogmatique, c'est-à-dire comme un renvoi au raisonnement d'une «autorité donnée pour indiscutable et immuable [...] L'argument dogmatique permet d'affirmer de façon péremptoire et contraint à admettre comme vraies certaines idées en raison de l'infaillibilité de la source » (Angenot 2013 : 217). Nguyễn Đình Cống (BVN, 25/11/2018) emploie un argument par l'analogie. Pour lui, la description des délits ressemble à celle « d'un non-voyant sans cerveau qui a seulement entendu parler d'un tableau mais veut expliquer aux autres ce tableau. » En référence à la fable Les aveugles et l'éléphant (six aveugles, en touchant différentes parties de l'éléphant, donnent des descriptions différentes de l'animal), l'auteur considère ceux qui reprennent fidèlement les accusations comme des aveugles sans aucune imagination ni réflexion. Pourtant, ils ont la prétention d'éclairer les autres sur une chose dont ils n'ont pas la moindre idée, autrement dit, ils ignorent même leur ignorance. Ainsi, les intellectuels vietnamiens estiment que l'esprit dogmatique, la cécité et la surdité du PCV rendent impossible tout dialogue.

\subsection{Le discours hostile disqualifiant}

Le discours disqualifiant transforme le discours de l'Autre en "discours second" (Authier-Revuz 1992 : 38) au travers des modalisations qui accentuent le rapport hostile du locuteur-rapportant à cet Autre. Dans cette polémique, il n'y a pas d'échange dialogal face-à-face entre les deux camps, mais une omniprésence des discours rapportés sans citer les sources. C'est pour cela que nous traiterons ici de la «modalisation autonymique» (ibid.: 39). L'autonymie désigne des mots ou des fragments du discours de l'Autre qui renvoient à eux-mêmes: ils sont donc autosuffisants et irremplaçables. Dans le discours disqualifiant, les signes autonymiques se réfèrent à des lieux communs (stéréotypes, clichés, slogans, idées reçues) et font souvent l'objet d'une dénégation. La modalisation autonymique vise donc à accentuer le décalage entre le discours autonymique rapporté et la réalité, à travers plusieurs formes de dénégation : ironie, allusion, métaphore, détournement ou rejet... Les intellectuels contestataires recourent régulièrement à la modalisation autonymique, sur plusieurs modes différents, lorsqu'ils rapportent les formules d'auto- 
transformation et d'auto-évolution. Nous présentons ici trois modes: argumentatif, émotif et ludique.

En réaction à la sanction contre Chu Hảo, quelques personnalités de renom ont déclaré quitter le parti. Dans sa déclaration, l'écrivain Nguyên Ngọc (BVN, 27/10/2018) rapporte la formule d'«auto-évolution" sous la forme d'un détournement de son usage initial : " Je trouve que depuis des années le Parti s'éloigne de l'idéal des débuts, "auto-évolue" et devient une organisation autoritaire qui trahit son peuple et détruit son pays ». Par un procédé de rétorsion, Nguyên Ngọc maintient le sens initial de la formule en la retournant contre le parti. Il le fait sur le mode argumentatif en s'appuyant sur l'argument d'autorité : sa décision de quitter le parti est justifiée par la déviation idéologique de cette organisation politique. Cette prise de décision provient de sa fidélité à l'idéal communiste et de l'impératif moral en tant que membre du parti : ce n'est donc pas lui mais le parti qui a trahi ses membres et le peuple. En se positionnant en tant que insider - posture lui permettant d'appuyer la légitimité de sa parole, Nguyên Ngọc dénonce le décalage entre la parole du PCV et son hypocrisie effective.

Dans l'exemple suivant, la modalisation autonymique se déploie sur le mode émotif à travers l'usage d'une métaphore :

Hoàng Dũng, $B V N, 26 / 10 / 2018$ : La sanction contre Chu Hảo est un message clair adressé aux intellectuels patriotes: "Vous ne pouvez penser, parler et agir que selon le rail établi. Soyez sages! Si vous déviez de ce rail, l'épée de Damoclès de l'auto-évolution et de l'auto-transformation tranchera vos cous ».

«L'épée de Damoclès » représente la situation permanente de danger qui plane sur la vie des intellectuels et dénonce de ce fait un argument par la peur dans le discours de l'Autre; la sanction contre Chu Hảo est ainsi un avertissement. Les formules fonctionnent ici comme un discours illocutoire de menace. L'essentiel du contrôle autoritaire réside dans la frontière floue qui existe entre ce qui est interdit et ce qui est autorisé ; cette ambiguïté permet d'intervenir de manière arbitraire dans les actions des citoyens. La force dissuasive de ces formules provient justement de leur sens flou: toute pensée, toute parole et toute action pourraient être accusées, à un moment ou à un autre, au titre de ces délits; ainsi, elle tue dans l'œuf toutes les possibilités de penser, de parler ou d'agir. Ces formules servent donc d'arme de dissuasion pour faire taire des intellectuels.

Dans le troisième exemple, le locuteur rapporte de manière ironique les formules sur le mode ludique :

Lưu Trọng Văn, Facebook, 26 octobre 2018 : Objectivement, le type doit reconnaître que cette sanction est... juste. Le délit du bonhomme est clairement l'autoévolution. Impossible d'objecter. [...] Il auto-évolue lorsqu'il demanda à son parti de reprendre l'ancien nom de Parti travailliste, comme à l'époque du vieux Hô. C'est le délit contre le règlement du parti, immanquable. [...] Ce n'est pas encore fini. La constitution en vigueur réglemente la direction unique du parti dans l'article 4, lui, en auto-évoluant, revendique la suppression de cet article, c'est clairement une dégénérescence politique, quoi d'autre?

Lưu Trọng Văn emploie à dessein une bi-vocalité qui mélange sa voix narrative (le type) et le discours du parti (auto-évolution, auto-transformation, dégénérescence politique) en simulant une complicité entre le narrateur et le discours de l'Autre (cette décision de sanction est juste). Cette bi-vocalité est effectuée sur un ton ironique, visant à tourner à contre-sens ces formules (antiphrase). Les fautes décrites relèvent de facto des recommandations des intellectuels réformistes pour réformer le système politique 
(changement du nom du parti, suppression de l'article 4 réglementant la direction unique du PCV). En s'alignant avec ces recommandations, le sens des formules est totalement inversé : le jugement négatif, du point de vue des autorités, s'efface en faveur du sens positif d'une évolution de la société, du point de vue des réformistes.

Quant aux médias orthodoxes, la modalisation autonymique se déploie à travers des citations du discours de l'Autre qui sont accompagnées de gloses méprisantes et sans évoquer la source :

L'Armée du peuple, 11/11/2018: [...] certains articles supposent que monsieur Chu Hảo est un « intellectuel légitime » qui « reviendra au peuple » après sa sanction pour mieux contribuer au développement du pays «en tant qu'homme libre » [...] certaines personnes autoproclamées progressistes et activistes pour la démocratie [...] disaient que la sanction à l'encontre de monsieur Chu Hảo, usant des « raisons calomnieuses ", a pour but d' " attaquer les intellectuels patriotes ayant du talent et du cœur » et ont ainsi obligé « des intellectuels à tourner le dos ».

Le média rapporte les énoncés de certaines personnalités qui ont été exprimés dans les médias étrangers et sur les réseaux sociaux. La modalisation autonymique, qui insère ces énoncés soit sous forme marquée (entre guillemets), soit sous forme libre avec glose ("autoproclamées»), vise à démontrer un décalage entre les appellations ("intellectuel légitime, homme libre, progressistes, activistes») et la réalité. Ce discours disqualifiant est accentué par l'anonymisation des sources («certains avis, certaines personnes, certains articles») - une forme de confiscation de la parole de l'Autre. Mais un lecteur bien informé pourrait facilement identifier les auteurs de ces énoncés qui ne sont rien d'autre que les intellectuels ayant déclaré quitter le parti. Face à ces intellectuels de l'establishment, les médias officiels jouent le jeu d'absenceprésence : en évitant de mentionner leurs noms propres, ils rapportent leurs énoncés explicites accompagnés de gloses mordantes; la modalisation autonymique fait de ces énoncés l'incarnation même de leurs auteurs - ces derniers sont donc la cible implicite de ces propos méprisants.

\subsection{Le discours de réfutation}

31 Le discours antagoniste vise à réfuter le discours de l'Autre ou à discréditer l'adversaire en remettant en cause la légitimité de sa parole. C'est l'image de l'Autre que le discours de réfutation donne à voir et qui participe à délégitimer son discours.

Dans le langage communiste, il existe plusieurs termes génériques réservés à l'ennemi : "forces hostiles, réactionnaires, contre-révolutionnaires, opportunistes politiques, éléments subversifs, organisations et individus mal intentionnés». Ces termes, employés systématiquement par les médias de l'État-parti, relèvent d'un champ lexical institutionnalisé : ils sont objectivés car légitimés par le discours du pouvoir.

Or, face à Chu Hảo, un ancien dirigeant et un intellectuel organique du parti, les médias officiels ne pourraient pas employer les termes réservés à l'ennemi du dehors. Ils ont donc recours à une pratique classique du communisme dans le traitement de l'ennemi du dedans : la critique et l'autocritique. Pour discipliner un membre qui a commis des erreurs, le Parti met en place des séances de critique collective dans lesquelles les camarades le critiquent d'une seule voix; la critique collective est complétée par l'autocritique de l'accusé. Le rapport dialectique et inextricable de critique et d'autocritique a pour but de réajuster le positionnement politique des membres, de renforcer l'unité et de conserver la pureté du Parti (Dittmer 1973). 

des discours rapportés, les critiques des intellectuels du régime contre Chu Hảo. Le choix des voix intellectuelles n'est pas anodin mais vise à répliquer à l'argument d'« attaque contre les intellectuels " par les contestataires. Or, ces intellectuels sont des membres dévoués et fidèles du parti : leurs voix s'expriment en total accord avec l'argument d'autorité. L'article intitulé "Sanctionner sévèrement afin de renforcer la discipline et l'ordre du parti et la loi de l'État» du journal L'Armée du peuple $(27 / 10 / 2018)$ et les trois reportages de la télévision vietnamienne VTV (29 octobre, 15 et 16 novembre 2018) sont à cet égard représentatifs. Ils rapportent les paroles des militaires et des dirigeants des organismes du PCV (Comité, Bureau central de la propagande et de l'instruction, Comité central des ressources humaines, Front de la patrie) mais aussi celles des vétérans, des cadres retraités et des théoriciens doctrinaires (Institut de l'histoire du parti, Institut de la construction du parti, Institut de la politique nationale de Ho Chi Minh). Ces locuteurs, présentés par les médias comme des intellectuels, parlent d'une même voix dans la scène de la «coénonciation » (Moirand 2002 : 179) lorsqu'ils critiquent Chu Hảo et ses délits :

L'Armée du Peuple, 27/10/2018: la perte de soi-même, affaiblissement de l'idéal révolutionnaire, orgueilleux, égoïste, outrecuidant, dégénéré, aliéné, membre qui a enfreint le règlement interne du parti [...]; VTV, 29/10, 15-16/11/2018: intellectuel du parti ayant un point de vue erroné, membre immérité, pensée aberrante, manque d'esprit constructif, opportuniste, membre dégénéré et corrompu, arrogant, mécontent [...].

Un fin connaisseur du langage communiste pourrait identifier dans ces mots non les attaques ad hominem mais les descriptions stéréotypées des symptômes de la "dégénérescence de la morale révolutionnaire ». Emprunté aux communistes chinois" le concept de morale révolutionnaire vise à anéantir l'égoïsme et l'individualisme - les deux symptômes de la mentalité bourgeoise. Ces dernières années, le PCV lance un reproche ferme contre "l'orgueil communiste » qu'il estime exister chez certains hauts dirigeants : selon lui, cet orgueil est caractéristique des "officiants méritants », ceux qui critiquent le parti et se mettent au-dessus de sa discipline interne, dont Chu Hảo est un exemple. Selon L'Armée du peuple (article du 10 octobre 2019) et le JT vietnamien (le 20 octobre 2019), son cas est révélateur de « la maladie des officiants méritants. »

Lorsque cette critique collective est médiatisée par la télévision, elle semblerait produire un effet de communion plutôt que celui d'argumentation. Dans une autre étude (Nguyen-Pochan 2018), nous avons examiné l'« espace de communion » dont la visée est de produire des effets de fusion et de convergence plutôt que de divergence et de désunion chez les participants. Dans ce cas précis, les voix orchestrées décrivent les délits de Chu Hảo non seulement comme un comportement inapproprié à l'égard des règles du parti mais aussi comme une "fausse conscience " - "une aliénation de la conscience, renvoie aussi à des manières de penser et à des mentalités étrangères à la “santé" cognitive » (Angenot $2008: 33$ ), que l'on doit éliminer du corps «sain » comme une "expurgation nécessaire afin de garder la pureté du parti » (L'Armée du Peuple, 11/11/2018). Ces discours attribuent ainsi à l'accusé le statut de profanateur qui a souillé la pureté du corps collectif et mérite d'être excommunié.

Dans le camp adverse, le discours de réfutation est beaucoup plus chargé d'émotion, marqué par l'usage de mots forts voire violents. La liste des qualitatifs péjoratifs réservés au PCV est inépuisable. On peut les répartir ici en les quatre catégories nonexhaustives suivantes : ignorance (mentalité archaïque, arriéré, stupide, obscurantiste), 
cécité (aveugle, dogmatique, perdre la tête, fanatique), pourriture (corrompu, trahison de la nation, honte, humiliation, cynique) et brutalité (totalitaire, dictateur, empereur rouge ${ }^{9}$ ).

L'écrivain Nguyên Ngọc (BVN, 27/10/2018) dénonce le Parti comme " une organisation immorale» tandis que Chu Hảo $(B V N, 29 / 10 / 2018)$ le traite d'« organisation sans aucune légitimité ». Ces propos, exprimés dans leurs déclarations de séparation du parti, accusent le PCV de dégénérescence et d'égoïsme. En rappelant leur contribution à la libération nationale et au développement du pays, ces intellectuels insider accusent implicitement le Parti d'ingratitude et de volte-face lorsqu'il attaque ses anciens " compagnons de route." Chu Hảo décrit les accusations du comité comme des manœuvres "sournoises", sous-entendant par là une attaque personnelle dégradante. Aux yeux de ces " officiants méritants ", le Parti n'a plus aucune légitimité en tant que force dirigeante. Phạm Đình Trọng (BVN, 27/11/2018), écrivain de l'Armée qui a quitté le parti bien avant cette affaire, compare la sanction contre Chu Hảo avec « la réforme agraire dans la campagne du Nord Vietnam au milieu du XXe siècle», en rappelant l'atrocité de cette réforme ${ }^{10}$. Cette analogie excessive réaffirme son rejet catégorique du rôle du parti.

Les intellectuels du parti, quant à eux, sont traités par les contestataires comme " plumes serviles, pseudo-intellectuels, instruments du pouvoir, intellectuels parasites et instrumentalisés, marionnettes, fidèles aveugles ». Plusieurs auteurs citent le propos de Mao Tsé Tong «Les intellectuels sont les excréments humains» afin de critiquer l'attitude anti-intellectuelle du PCV ou de le retourner contre ses propres intellectuels : «Ce n'est peut-être pas faux. Les intellectuels et "plumes serviles” tels que Nhị Lê, Vũ Mão, sont-ils différents des excréments humains ?» (Bùi Quang Vơm, Journaliste citoyen, 30 octobre 2018). Sur sa page Facebook Lão Tạ (16/11/2018), l'écrivain Tạ Duy Anh a commenté la mise en scène des voix rapportées par le journal télévisé (16 novembre 2018) comme suit :

Tant d'énergie et de temps pour transformer un banni du parti en phénix alors que les soldats fidèles et les protecteurs du régime, bouches pleines de bave, ressemblent à un poulet abattu. Quelle pitié !

40 Tạ Duy Anh feint de critiquer la mise en scène télévisée qu'il estime très maladroite : au lieu de produire les effets visés, elle n'a généré que des effets contre-productifs. Sa feintise rhétorique vise à ridiculiser l'effort des médias officiels pour changer la donne : cela s'avère impossible et lamentable à ses yeux dans un affrontement « asymétrique » qui oppose Chu Hảo, « un grand intellectuel qui suscite l'engouement de dizaines de milliers de personnes » à quelques «visages abrutis » dont la voix n'a " aucune crédibilité ».

\subsection{Le processus dialogique}

41 Du côté du PCV, le processus dialogique peut être observé dans les discours du secrétaire général Nguyễn Phú Trọng rapporté par les médias officiels :

Le Peuple, 29/10/2018 : Nous sommes humains, ce n'est pas pour notre plaisir que nous sanctionnons nos camarades, au contraire, cela est douloureux. Mais pour le bien de tous [...] nous sommes obligés de discipliner quelques personnes afin d'en sauver des milliers d'autres.

Le Peuple a inséré ce discours, énoncé le 25 février 2017, dans son article comme appui pour arguer que le traitement de Chu Hảo n'est guère personnel mais simplement un 
exemple de la discipline du parti. Énoncé lors d'une réunion nationale du PCV, ce discours semble vouloir justifier la sanction contre «quelques personnes " comme une nécessité pour assurer l'ordre du Parti, et ce, malgré «notre douleur ». L'expression «nos camarades" suggère que Nguyễn Phú Trọng les considère toujours comme membres du parti. Mais le fait qu'il s'abstient de mentionner leurs noms propres apparaît comme une manière de les présenter comme des exemples de la discipline donnés à voir aux autres et non comme des attaques personnelles.

Nguyễn Phú Trọng rappelle cette discipline impersonnelle du parti à une autre occasion, lors de sa rencontre avec les membres d'une cellule locale, cette fois-ci en pleine affaire Chu Hảo :

L'auto-évolution et l'auto-transformation existent dans chaque personne, elles nous transforment en un autre sans que nous nous en rendions compte, elles orientent notre chemin vers des directions compliquées [...] Comme cela s'est déjà dit: discipliner quelques personnes afin d'en sauver des milliers d'autres [...] La dégénérescence sur le plan politique est plus dangereuse que sur le plan économique (discours rapporté, Le Travailleur, 24/11/2018).

Il estime que l'auto-évolution et de l'auto-transformation sont particulièrement dangereuses sur le plan idéologique, et ce d'autant plus qu'elles sont «humaines » et « insidieuses ». Cette approche semblerait redéfinir la lutte contre l'ennemi du dedans : il ne s'agit plus de la sanction contre les membres accusés, mais d'un combat intérieur que chaque membre doit mener en permanence pour résister aux influences néfastes des pensées erronées et renforcer un positionnement idéologique correct. La complexité de ce nouveau combat oblige le parti à appliquer des mesures de discipline fermes pour sa survie politique. Le discours du secrétaire général est doté ici d'un « dialogisme interlocutif » (Moirand 2002), car il s'adresse aussi à ses "camarades » ayant subi la sanction. Ce positionnement ambigu induirait l'idée que la sanction porte plutôt sur la forme que sur le fond.

Du côté des intellectuels contestataires, leur discours dialogique se révèle dans leur positionnement insider et leur lien organique avec le régime, par exemple :

Nguyễn Trung (Viet-studies, 27/10/2018) : Notre pays actuel est confronté à des défis périlleux sans précédent depuis la Réunification il y a 43 ans [...] Je me demande [...] s'il n'y a pas d'autres choses plus importantes à faire [...] que diviser la solidarité nationale et affaiblir la force interne comme la sanction contre le professeur $\mathrm{Chu}$ Hảo ? Le comité est-il insensible au point de ne pas apercevoir ces défis vitaux et de décider une telle sanction?

Si le discours du secrétaire général inscrit la sanction dans la lutte actuelle du parti contre les ennemis du dedans, celui de Nguyễn Trung l'insère dans un temps plus long : celui du Vietnam réunifié depuis 1975. Dans cette perspective, il impute au PCV la responsabilité d'affaiblir la force nationale qui pourrait entraîner des conséquences néfastes pour le pays dans le long terme. Écrit à la première personne et en s'adressant directement au comité, ce discours semble prendre la forme d'une critique interne (entre Nous), plutôt que celle venue de l'extérieur, qui lui permettrait d'entretenir le processus dialogique avec les autorités.

\subsection{Les voix dissidentes}

Si la polarisation est souvent mise en avant dans les études de la polémique, la fracture au sein d'un même camp est peu examinée. La logique polémique veut que les locuteurs 
du même camp s'entendent et se serrent les coudes. Or, dans le cas des intellectuels contestataires, leur camp n'est qu'une " communauté transitoire " (Grinshpun 2018: 78) qui rassemble temporairement les insider et les dissidents dans le temps court d'un événement et autour d'un point de vue partagé : leur mécontentement envers le PCV. Cependant, la fracture extra-polémique persiste, bien qu'elle soit moins visible dans cette période qu'en temps normal. Les dissidents sont nombreux à participer à la polémique et à soutenir Chu Hảo qui représente à leurs yeux, en ce moment précis, le symbole du milieu intellectuel humilié par le Parti. Mais une minorité des voix dissidentes cherche à rappeler au public le lien qui unit Chu Hảo et le régime : bien qu'il ait participé à plusieurs activités d'opposition, il maintient toujours son statut officiel (ses fonctions dans les institutions publiques), son statut d'adhérent du parti et poursuit le dialogue avec le PCV. Ces voix dissidentes adressent leur reproche à Chu Hảo plutôt qu'au Parti, mais la gravité de leurs reproches est différente d'un auteur à l'autre en fonction du rapport qu'ils entretiennent avec le destinataire. Dans les exemples suivants, elles s'expriment sous la forme d'une critique aimable :

Hoàng Quốc Hải, BVN, 4/11/2018: Chu Hảo, vous avez fait une erreur aimable, vous avez eu un espoir illusoire qui vous a conduit à vos "échecs ", quand vous pensiez que notre pays devrait avoir un "souverain éclairé » comme le Japon sous l'ère de Meiji [...].

Huỳnh Anh Tú, Journaliste citoyen, 27/10/2018 : Je pense à Hoàng Minh Chính, Trần Độ, Nguyễn Thanh Giang, Lê Hồng Hà, Vũ Cao Quận... [...] qui n'ont pas seulement quitté le parti, ils ont aussi consacré toute leur vie à contester le parti et lutter pour la liberté et la démocratie du pays [...] Si monsieur Chu Hảo n'était pas sanctionné, quitterait-il son parti?

Hoàng Quốc Hải dénonce, par sa critique, l'erreur de poursuivre le dialogue avec un pouvoir qui est loin d'être un « souverain éclairé ». Quant à Huỳnh Anh Tú, il reproche de façon tacite à Chu Hảo d'avoir adopté une position confortable dans la lutte pour la liberté et la démocratie du pays par rapport à beaucoup d'autres militants qui n'ont pas choisi la même voie pour poursuivre leur combat.

Une voix dissidente comme celle de Phạm Thị Hoài est, pourtant, beaucoup plus dure à entendre, notamment lorsqu'elle parodie les accusations du comité et ironise la position insider de Chu Hảo :

Phạm Thị Hoài, Facebook, 31/10/2018 : En 2009, le Parti a failli lui donner un blâme mais est resté tolérant [...] « en prenant en compte des circonstances atténuantes telles que ses mérites, ses contributions et la sincérité du camarade ». En juin 2016, le Parti a encore doucement «invité le camarade à échanger, analyser, éclaircir et arrêter ses violations ". La troisième fois, en août 2016, c'est-à-dire deux mois après la rencontre, le camarade a commis une nouvelle fois une faute en rédigeant un article : «Il est venu le temps du dialogue ». Aujourd'hui, le Parti est contraint de reconnaître que "le symptôme de l'opportunisme politique du camarade était depuis longtemps en gestation ». Fin de quota. La quatrième fois sera impossible. La patience du Parti a ses limites. Mais jusqu'à la fin de l'article, le Parti appelle toujours monsieur Chu Hảo le camarade [...].

Phạm Thị Hoài dévoile le rapport organique qui existe entre Chu Hảo et le parti, en citant à plusieurs reprises le mot "camarade " sur un ton ironique. Son point de vue sur la posture insider de Chu Hảo demeure inchangé et tranché malgré la sanction qu'il subit : l'écrivaine l'a fortement critiqué en 2012 lors d'un débat autour de son article « Il est venu le temps du dialogue ", et lui attribua alors l'appellation d'« opposant fidèle ». Moyennant la mise en scène d'un rapport amour-haine, l'écrivaine présente l'affaire comme un tour de passe-passe entre le Parti et ses membres. Elle semble suggérer 
qu'une telle comédie ne mériterait pas autant de bruit et de commentaires de la part des intellectuels.

\section{Conclusion : les enjeux discursif et politique de la polémique}

51 Dans cette étude, la polémique dispose en apparence des modalités propres d'une polarisation en deux camps en contexte démocratique: les prises de position antagonistes, un rapport à l'Autre extrêmement hostile à travers l'usage des discours de réfutation et disqualifiant, la polyphonie des énoncés ou encore le recours à une autorité reconnaissable de tous; nous y trouvons aussi un processus dialogique relevant du discours polémique (Maingueneau 1984 :119). Mais dès que l'on examine de près ces modalités, la ressemblance s'avère moins évidente. D'une part, des modalisations des énoncés spécifient un type d'échange dialogique et non dialogal (rapport des discours sans citer les sources, usage des formes de dénégation, aucune mention des noms propres...). D'autre part, les camps adverses font appel à deux thésaurus de discours et à deux sources d'autorité parallèles qui transforment cette polémique en un véritable «dialogue de sourds » (Angenot $2008: 19$ ). Nous identifions également la fracture au sein du camp des contestataires qui sépare les intellectuels insider des dissidents. La polémique n'est ici qu'une surface émergée de tensions complexes et multipolaires qui s'expriment et se cristallisent dans une polarisation des opinions.

52 L'enjeu crucial de l'argumentation polémique réside cependant non pas dans les définitions et les positionnements des deux camps adverses, mais dans une coexistence des discours antagonistes. Dans le contexte autoritaire, il relève d'un enjeu politique de taille : la visibilité de la polémique dans les espaces numériques donne à voir un pas, sinon déterminant, au moins significatif, vers la démocratisation de l'espace public.

En effet, on ne peut comparer cette "attaque contre les intellectuels" avec les campagnes d'élimination des intellectuels commises par le passé - auxquelles les contestataires se réfèrent pourtant régulièrement, telles que les lourdes sanctions contre les artistes contestataires des revues littéraires Humanisme et Belles Euvres pendant les années 50 ou contre les intellectuels accusés d'être des "révisionnistes contre le parti » pendant les années 60 . Pour ces accusés, la possibilité de polémiquer n'existait même pas. Il était impensable qu'ils puissent tenir les mêmes propos que leurs pairs d'aujourd'hui. La visibilité même de la polémique suffit à montrer que la tension est gérée par une guerre des mots et non par la coercition ou la prison.

Nous tenons toutefois à nuancer cet optimisme car les espaces publics numériques demeurent strictement surveillés, de nombreux sites d'information, y compris ceux qui sont l'objet de cette étude, sont régulièrement piratés ou bloqués par des pare feux, leurs auteurs ont subi des harcèlements de la police. En réalité, leur information est lue par une minorité d'internautes provenant de l'intelligentsia vietnamienne. Pourtant, la visibilité de la polémique est révélatrice à la fois d'un nouveau mode de gestion des tensions par l'État-parti et d'une nouvelle posture des intellectuels contestataires: ceux-ci parlent désormais d'égal-à-égal avec le pouvoir dans l'espace discursif. Nous rejoignons ici la conception pragmatique de Burger (2011) selon laquelle la guerre des 
mots relève plutôt de la négociation des rapports de force dans les espaces public et politique que du dialogue des sourds sans but précis.

\section{BIBLIOGRAPHY}

Amossy, Ruth. 2014. Apologie de la polémique (Paris : PUF, coll. L'interrogation philosophique)

Angenot, Marc. 2008. Dialogues de sourds. Traité de rhétorique antilogique (Paris : Fayard)

Angenot, Marc. 2013. Rhétorique de la confiance et de l'autorité (Montréal : Discours social, vol. 44)

Authier-Revuz, Jacqueline. 1992. « Repères dans le champ du discours rapporté », L'information grammaticale $55,38-42$

Arendt, Hannah. 2002 (1951). Les origines du totalitarisme (Paris : Gallimard)

Burger, Marcel. 2011. « Une caractérisation praxéologique du désaccord polémique : ce qu'informer dans les médias veut dire », SEMEN 31, 61-80

Cain, Geoffrey. 2013. "Kill One to Warn One Hundred: The Politics of Press Censorship in Vietnam", The International Journal of Press/Politics XX(X), 1-23

Dittmer, L., 1973, “The Structural Evolution of Criticism and Self-Criticism”, China Quartely 56, 708-729

Fiskesjö, Magnus. 2017. “The Return of the Show Trial: China's Televised 'Confession'“, The AsiaPacific Journal, vol.15 (13), 1-34

Guillemot, François. 2016. «Viet Nam : révolution en devenir, langage totalitaire : questions sans réponse ", Indomémoires : www.indomemoires.hypotheses.org/22007

Grinshpun, Yana. 2018. " Mémoire et communauté transitoire », H. Barthelmebs-Raguin, G. Komut-Thilloy, J. M. Lopez-Munoz, S. Marnette \& L. Rosier (éds). Le discours rapporté. Temporalité, histoire, mémoire et patrimoine discursif (Paris : Classiques Garnier), 77-88

Hữu, Thọ. 1998. Le métier des gens de presse (Hanoi : Education).

Krieg-Planque, Alice. 2009. La notion de « formule » en analyse du discours. Cadre théorique et méthodologique (Besançon : Presses Universitaires de Franche-Comté).

London, Jonathan. 2014. "Politics in Contemporary Vietnam", Jonathan London (eds), Politics in Contemporary Vietnam. Party, State and Authority Relations (UK: Palgrave, McMillan), 1-20

Maingueneau, Dominique. 1984. Genèse du discours (Bruxelles : Mardaga)

Moirand, Sophie. 2002. « Dialogisme », Patrick Charaudeau, Dominique Maingueneau (eds), Dictionnaire d'analyse du discours (Paris : Seuil), 175-178

Nguyen-Pochan, Thi Thanh Phuong. 2018. « La construction de sens de l'événement par le Journal télévisé vietnamien. Le cas des manifestations ouvrières antichinoises ", Études de communication 51, 207-223

Papin, Philippe, Laurent Passicousset. 2010. Vivre avec les Vietnamiens (Paris : L'Archipel). 
Phạm, Thị Hoài. 2012. «L'optimisme sans limite », version vietnamienne de la BBC : https:// www.bbc.com/vietnamese/mobile/forum/2012/01/120117_phamthihoai_vn_intelligentsia.shtml Phạm, Thị Hoài. 2009. « La dignité des intellectuels vietnamiens », danluan, 18 mars : https:// www.danluan.org/tin-tuc/20090318/pham-thi-hoai-tu-cach-tri-thuc-viet-nam

Phan, Văn Kiền. 2015. La critique sociale de la presse vietnamien à travers quelques événements remarquables (Hanoi : Information et communication)

Plantin, Christian. 1996. L'argumentation (Paris : Seuil)

Thayer, C. A. 2006. "Political Dissent and Political Reform in Vietnam 1997-2002", C. Derichs \& T. Heberer (eds), The Power of Ideas. Intellectual Input and Political Change in East and Southeast Asia (Copenhagen: NIAS Press): 115-132

Thayer, Carlyle A. 1999. "Vietnamese Foreign Policy: Multilateralism and the Threat of Peaceful Evolution”, Carlyle A. Thayer (ed.). Vietnamese Foreign Policy in Transition (Singapore: Institute of Southeast Asia Studies), 1-24

Trần, Đăng Tuấn. 2006. La critique sociale (les questions posées par la vie sociale) (Danang : Đà Nẵng)

\section{NOTES}

1. Nous remercions vivement Ruth Amossy pour ses commentaires et suggestions qui nous ont permis de reformuler et approfondir la problématique de cette recherche.

2. L'appellation de l'État-parti désigne l'accaparement de l'État par le PCV.

3. www.boxitvn.blogspot.com ; www.danlambaovn.blogspot.com; www.baotiengdan.com; et www.viet-studies.net

4. Ces réformes furent lancées par le PCV à la suite de son Sixième Congrès national en 1986.

5. Depuis 1945 avec la fondation de la République démocratique du Vietnam

6. Résolution du Comité central $n^{\circ} 4-\mathrm{NQ} / \mathrm{TW}$, le 30 octobre 2016, concernant « les missions pour renforcer le parti, dissuader et repousser les symptômes de la dégénérescence de la pensée politique, de la morale et du mode de vie et ceux de l'"auto-transformation" et de l"'autoévolution" ".

7. Nous traduisons les citations vietnamiennes en langue française.

8. Notamment à Liu Shaoqi, auteur de Pour être un bon communiste (1939), cf.: https:// www.marxists.org/francais/general/liu-shaoqi/works/1939/07/pour_etre/index.htm

9. Ce concept renvoie à l'idée d'un pouvoir absolu ou d'un despotisme communiste.

10. Dans la réforme agraire (1953-1956), les dénonciations collectives des villageois furent conçues comme un instrument politique du régime pour éliminer les ennemis de la révolution ; pour les dénonciateurs eux-mêmes, ils y trouvèrent un moyen légitime pour se venger et satisfaire leur désir haineux et leur jalousie vis-à-vis de leur propre famille ou de leurs voisins.

\section{ABSTRACTS}

Through an analysis of a controversy about the accusations of "self-transformation" and "selfevolution", formulated by the Vietnamese Communist Party (VCP) against Chu Hảo, an 
intellectual-member of the VCP, this paper aims to test the hypothesis whereby the existence of controversy would make possible coexistence in discord and thus democratize the public space in an authoritarian regime. Our analysis is based on the media coverage of this case produced over the last three months of 2018 and by two different sources: the Party-State's official media (11 information occurrences) and social media (23 information occurrences from four websites and 8 Facebook's entries). We will shed light on the procedures of polarization and the dialogical process that organize the co-presence of antagonistic discourses in the public space, but also on the facture that emerges from the camp of protesting intellectuals.

Par le biais d'une analyse de la polémique autour des accusations d'«auto-transformation » et d'« auto-évolution », formulées par le Parti communiste vietnamien (PCV) à l'encontre de Chu Hảo - un intellectuel-membre du PCV, cet article cherche à vérifier l'hypothèse selon laquelle la polémique rendrait possible une coexistence dans le dissensus et démocratiserait ainsi l'espace public en régime autoritaire. Notre analyse s'appuiera sur un corpus constitué des occurrences d'information couvrant cette affaire pendant les trois derniers mois de 2018 et qui sont issues de deux sources : onze occurrences issues des médias officiels de l'État et du PCV et 23 occurrences issus des médias et réseaux sociaux sur Internet. Nous éclaircirons les procédures de polarisation et le processus dialogique qui organisent la coprésence des discours antagonistes dans l'espace public, mais aussi la fracture issue du camp des intellectuels contestataires.

INDEX

Mots-clés: autoritarisme vietnamien, démocratisation. Internet, médias, polémique

Keywords: controversy, democratization, Internet, media, Vietnamese authoritarianism

\section{AUTHOR}

\section{THI THANH PHUONG NGUYEN-POCHAN}

CEMTI (Université Paris 8) ; CASE (EHESS) ; Université de Lille 